\title{
Author Correction: Liver macrophages regulate systemic metabolism through non-inflammatory factors
}

Cecilia Morgantini (D, Jennifer Jager, Xidan Li, Laura Levi, Valerio Azzimato, André Sulen, Emelie Barreby, Connie Xu, Michaela Tencerova, Erik Näslund, Chanchal Kumar, Francisco Verdeguer, Sara Straniero, Kjell Hultenby, Niklas K. Björkström (D), Ewa Ellis, Mikael Rydén D, Claudia Kutter, Tracey Hurrell, Volker M. Lauschke, Jeremie Boucher, Aleš Tomčala, Gabriela Krejčová, Adam Bajgar and Myriam Aouadi (D)

Correction to: Nature Metabolism https://doi.org/10.1038/s42255-019-0044-9, published online 25 March 2019.

In the version of this article initially published, in Fig. 4c, the $y$-axis units read 'mg/dl' but should have read 'percent baseline'. The error has been corrected in the HTML and PDF versions of the article.

Published online: 19 January 2021

https://doi.org/10.1038/s42255-021-00343-5

(C) The Author(s), under exclusive licence to Springer Nature Limited 2021 